\title{
A Simulation-Assisted Non-destructive Approach for Permittivity Measurement Using an Open-Ended Microwave Waveguide
}

\author{
Zhen $\mathrm{Li}^{1} \cdot$ Arthur Haigh ${ }^{2} \cdot$ Constantinos Soutis ${ }^{1} \cdot$ Andrew Gibson ${ }^{3} \cdot$ Robin Sloan ${ }^{2}$
}

Received: 9 December 2017 / Accepted: 8 May 2018 / Published online: 17 May 2018

(c) The Author(s) 2018

\begin{abstract}
A new convenient and non-destructive permittivity measurement method is presented. No physical cut of specimens is needed here for material characterisation. In the setup, the material under test is placed in the near-field region of a microwave openended waveguide. An electromagnetic model of the setup is built in the Computer Simulation Technology simulation software. Employing optimisation, the permittivity is obtained from the measured reflection coefficients $S_{11}$. Using the same technique, the effect of the model size is investigated that could reduce the modelling effort for large structures. The efficiency of a traditional method (i.e., Newton) and an intelligent algorithm (i.e. particle swarm optimisation) for permittivity calculation is thoroughly studied and compared. The proposed methodology is validated by experimental data. It is demonstrated that the proposed method can provide more accurate permittivity results than the intrusive in-waveguide measurement. The proposed methodology can contribute to electromagnetic analysis, thickness measurement and non-destructive evaluation.
\end{abstract}

Keywords Electric permittivity $\cdot$ Electromagnetic simulation $\cdot$ Optimisation $\cdot$ Microwave $\cdot$ Non-destructive evaluation

\section{Introduction}

Microwaves are commonly used for telecommunications and food processing, while extensive attention has been given to their potential in material characterisation (e.g., porosity evaluation [1,2] and moisture measurement [3]) and non-destructive testing (e.g., corrosion detection [4], crack detection [5-7], thickness variation [8], delamination detection [9,10] and impact damage inspection [11-14]). Microwaves propagate in air and dielectric materials with low attenuation. The electric permittivity is an intrinsic parameter of a material that describes the interaction with the electromagnetic field, and microwaves are highly sensitive to its variation. With the knowledge of the permittivity, the quality or condition of the material can be readily assessed.

Constantinos Soutis

constantinos.soutis@manchester.ac.uk

1 Aerospace Research Institute, The University of Manchester, Manchester M13 9PL, UK

2 School of Electrical and Electronic Engineering, The University of Manchester, Manchester M13 9PL, UK

3 Faculty of Science and Engineering, Manchester Metropolitan University, Manchester M1 5GD, UK
A number of microwave techniques have been used for permittivity measurement: resonance methods, transmission line technique, free space methods and open-ended rectangular waveguide/coaxial probe techniques. The resonance approach is inherently narrowband, and it requires careful sample preparation (spheres or cylinders required) and calibration [15]. For the transmission line technique, samples with the same inner dimensions of the waveguide need to be cut and perfectly fit within the waveguide. And the resolution of loss tangent measurements provided by this method is limited (typically \pm 0.01 ) [16]. In the free space method, the sample and the sample holder should be placed between two horn antennae, and special attention must be paid to the sample geometry and location. Hence, in these three methods, cutting and machining of test samples is required. Considering the time for contacting the mechanical workshop, task scheduling, actual machining work and delivery, the total time could be a couple of days. This kind of mechanical work is labour intensive and sample cutting from the examined component may not be permissible due to coatings [17] and could undermine structural integrity. Therefore, the open-ended method is well-suited for industrial non-destructive measurements. Compared with the coaxial open-ended probe, the open-ended rectangular waveguide is more capable for measurements of low-permittivity materials 
and anisotropic materials [16]. And the field region around the waveguide is larger than that around the coaxial probe, where electromagnetic fringe fields closely surround the tip of the probe [18].

For the open-ended waveguide method, permittivity calculation requires rigorous mathematical formulation of the electromagnetic field outside the waveguide flange. As the exact solution of Maxwell's equations for the field is not available, approximate models have been reported [1921]. In these models, a $2 \mathrm{D}$ problem is considered where the material space is commonly assumed infinite, and the analysis is limited to layered structures. Hence, the finite element method can be employed to provide more accurate description of the experimental arrangements and the electromagnetic field.

An appropriate optimisation method is also needed for permittivity extraction from one-port measurement data. The permittivity value used in the finite element modelling should to be optimised in order to produce the simulated reflection coefficients $S_{11}$ approximate the measured data. The optimisation techniques can be classified into two groups: iterative methods (e.g., Gauss-Newton, quasi-Newton, gradient and conjugate gradient) and heuristic methods (e.g., genetic algorithm (GA), particle swarm optimisation (PSO) and ant colony optimisation (ACO)). For the iterative methods, the gradient of the objective function usually needs to be calculated at each iteration, and the accuracy of the results depends on the selection of the initial estimation. However, for the heuristic algorithms, the gradient of the problem being optimised is not required. In addition, they have better global search abilities than traditional iterative methods.

In this paper, the non-destructive measurement of complex permittivity at microwave frequencies using an open-ended rectangular waveguide is presented. The experimental setup is modelled in the Computer Simulation Technology (CST) simulation software [22], and the input permittivity value is optimised until a converged solution is found. The calculation procedure is demonstrated by an example of a thin ceramic coated plate. The $S_{11}$ obtained from the forward simulation is used as the input for the inverse calculation. The iterative quasi-Newton method and the heuristic PSO method are employed, and the accuracy and efficiency of both methods are compared. The effect of the model size is investigated for large workpieces. Sample thickness is also evaluated using the same methodology. An experiment is conducted to measure the permittivity of the dielectric layer of a printed circuit board (PCB) with one-sided copper cladding. The experimental results obtained are compared with the manufacturer's data and results given by the microwave transmission line technique.

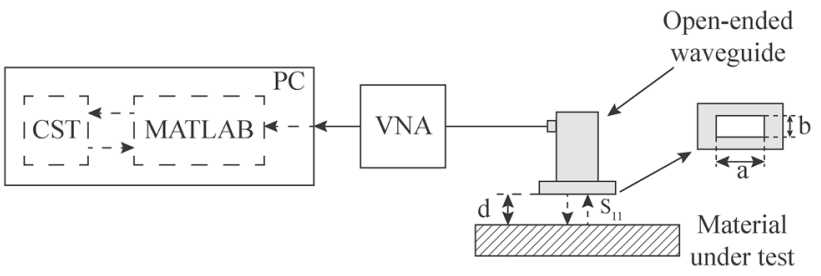

Fig. 1 Schematic diagram of the proposed simulation-assisted methodology for non-destructive permittivity measurement

\section{Definition of Permittivity}

The complex permittivity $\varepsilon$ can be described as:

$$
\begin{aligned}
\varepsilon & =\varepsilon_{0} \varepsilon_{r}=\varepsilon_{0}\left(\varepsilon_{r}^{\prime}-j \varepsilon_{r}^{\prime \prime}\right) \\
& =\varepsilon_{0} \varepsilon_{r}^{\prime}(1-j \tan \delta)
\end{aligned}
$$

where $\varepsilon_{0}=8.8542 \times 10^{-12} \mathrm{~F} / \mathrm{m}$ is the permittivity of free space, and $\varepsilon_{r}$ is the relative permittivity. The real part $\varepsilon^{\prime}{ }_{r}$, or dielectric constant, characterises the ability of a material to store the electric field energy. The imaginary part $\varepsilon_{\mathrm{r}}^{\prime \prime}$, or dielectric loss factor, reflects the ability of the material to dissipate the energy in the form of heat. $\varepsilon_{\mathrm{r}}^{\prime \prime}$ is positive due to energy conservation. In practice, the electrical behaviour is described by dielectric constant and loss tangent $\left(\tan \delta=\varepsilon_{\mathrm{r}}^{\prime \prime}\right.$ $\left./ \varepsilon^{\prime}{ }_{\mathrm{r}}\right)$.

\section{Non-destructive Measurement of Permittivity}

The schematic diagram of the proposed setup for complex permittivity measurement is presented in Fig. 1. The signal response of the material is measured with a Vector Network Analyser (VNA). The material test sample can be of an arbitrary shape, with or without a metal backplate and of a multi-layered dielectric construction with a layer of unknown permittivity or thickness. In the test, the sample should be placed within the near-field region of the waveguide, at a standoff distance $d$ of [23]:

$0 \leq d<2 a^{2} / \lambda$

where a is the broad inner dimension of the waveguide, and $\lambda$ is the wavelength of the incident electromagnetic field. For a frequency range, the minimum frequency is used for the estimation of the allowable standoff distance, so that the requirement can be met for all the frequencies used.

The flowchart that describes the permittivity calculation procedure is shown in Fig. 2. The reflection coefficients $S_{11}$ measured are imported to MATLAB ${ }^{\circledR}$ software. A 3D model of the measurement setup is built in the CST simulation tool 


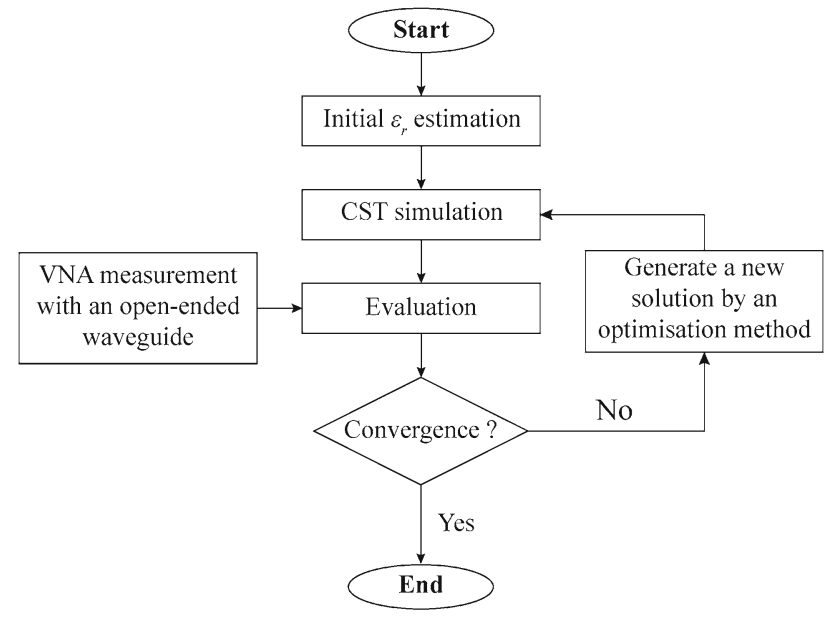

Fig. 2 Flowchart of the non-destructive permittivity calculation procedure using CST simulation and optimisation

using the parametric modelling technique. The MATLABCST interaction technique reported in $[9,10]$ is adopted here. MATLAB is utilised to control the simulation process via visual basic for application (VBA), which is the programming language in the CST environment. MATLAB calls the VBA programme to modify the permittivity, update the model and perform the electromagnetic calculation. The error function used for the evaluation of the solution is defined as: (ii) Check convergence: if $\left\|\nabla F_{\text {err }}\left(x_{k}\right)\right\|<\tau$, where $\tau$ is a pre-set tolerance.

(iii) The search direction is $\mathrm{h}_{k}=-\mathbf{H}_{\mathrm{k}} \quad \nabla \mathrm{F}_{\mathrm{err}}\left(x_{k}\right)$. Conduct a line search on the step size $\alpha$ from $\mathrm{x}_{k}$ in that direction. Take $\mathrm{x}_{k+1}=\mathrm{x}_{k}+\alpha \mathrm{h}_{k}$ to minimise $\mathrm{F}_{\mathrm{err}}\left(\mathrm{x}_{k}+\alpha \mathrm{h}_{k}\right)$. Here the bisection method is used for the 1D search of $\alpha$. The initial points of the 1D search are carefully selected to guarantee that all the solutions to be produced are physically acceptable $\left(\varepsilon_{\mathrm{r}}^{\prime} \geq 1\right.$ and $\tan \delta \geq 0$ for the present case). Repeat until the local maximum iteration $\mathrm{k}_{\max }^{\prime}$ is met.

(iv) Evaluate $\mathrm{F}_{\mathrm{err}}\left(\mathrm{x}_{k+1}\right)$ and $\nabla \mathrm{F}_{\mathrm{err}}\left(\mathrm{x}_{k+1}\right)$.

(v) Compute $\mathbf{H}_{k+1}$ by setting $\mathbf{H}_{k+1}=\mathbf{H}_{k}+\mathbf{U}_{k}$. Here the Broyden, Fletcher, Goldfarb and Shanno (BFGS) update expression of $\mathbf{U}_{k}[25]$ is employed.

$$
\begin{aligned}
\mathbf{U}_{k}= & \left(1+\frac{\Delta g_{k}^{T} \mathbf{H}_{k} \Delta g_{k}}{\Delta x_{k}^{T} \Delta g_{k}}\right) \frac{\Delta x_{k} \Delta x_{k}^{T}}{\Delta x_{k}^{T} \Delta g_{k}} \\
& -\frac{\mathbf{H}_{k} \Delta g_{k} \Delta x_{k}^{T}+\left(\mathbf{H}_{k} \Delta g_{k} \Delta x_{k}^{T}\right)^{T}}{\Delta x_{k}^{T} \Delta g_{k}}
\end{aligned}
$$

where $\Delta \mathrm{x}_{k}=\mathrm{x}_{k+1}-\mathrm{x}_{k}$ and $\Delta \mathrm{g}_{k}=\nabla \mathrm{F}_{\mathrm{err}}\left(\mathrm{x}_{k+1}\right)-$ $\nabla \mathrm{F}_{\mathrm{err}}\left(x_{k}\right)$.

(vi) Return to Step ii with $k=k+1$. Repeat until the convergence criteria or the maximum iteration $\mathrm{k}_{\max }$ is met.

$$
\begin{aligned}
& \mathrm{F}_{\mathrm{err}}\left(\varepsilon_{r}^{\prime}, \tan \delta\right) \\
& =\sqrt{\frac{1}{\mathrm{~N}_{f}} \sum_{i=1}^{\mathrm{N}_{f}}\left\{\left[\frac{\left|\mathrm{S}_{11, \text { meas }}\right|-\left|\mathrm{S}_{11, \mathrm{CST}}\right|}{\left|\mathrm{S}_{11, \text { meas }}\right|}\right]^{2}+\left[\frac{\angle \mathrm{S}_{11, \text { meas }}-\angle \mathrm{S}_{11, \mathrm{CST}}}{\angle \mathrm{S}_{11, \text { meas }}}\right]^{2}\right\}}
\end{aligned}
$$

where $\mathrm{N}_{f}$ is the number of the frequency sampling points. $\mathrm{S}_{11 \text {,meas }}$ and $\mathrm{S}_{11, C S T}$ are the measured and simulated $\mathrm{S}_{11}$, respectively. $\left|\mathrm{S}_{11}\right|$ and $\angle \mathrm{S}_{11}$ are the magnitude and phase of $\mathrm{S}_{11}$ in decibels $(\mathrm{dB})$ and degrees (deg), respectively.

If the convergence is not satisfied, a new solution is generated using an appropriate optimisation algorithm. In this work, two different methods are employed and discussed: the quasi-Newton method and the particle swarm optimisation.

\section{(1) Quasi-Newton method}

The quasi-Newton method is iterative, involving a series of line searches [24]. At each iteration, the function value and the derivative are computed. The main procedure is as follows:

(i) Set the iteration counter $k=1$ and an estimated permittivity $\mathbf{x}_{1}=\left[\varepsilon_{\mathrm{r}}^{\prime} \tan \delta\right]$. Initialise a matrix $\mathbf{H}_{1}=\mathbf{I}(2 \times 2$ identity matrix). Calculate the derivative of $\mathrm{F}_{\mathrm{err}}\left(x_{k}\right), \nabla$ $\mathrm{F}_{\mathrm{err}}\left(x_{k}\right)$, using finite-difference approximation.

\section{(2) Particle swarm optimisation (PSO)}

The PSO is a population-based algorithm, which was inspired by the social and cognitive behaviour of animals like fish schooling and bird flocking, adapting to the environment to find food sources [25]. A population (called a swarm) of candidate solutions (called particles) is used in the algorithm. The movements of these particles are guided by their own best-known positions as well as the knowledge gained by the swarm. For the permittivity calculation, the position of each particle with $\varepsilon^{\prime} r$ and $\tan \delta$ is in a 2D search space. The fundamental steps of the PSO are addressed:

Step 1 Initialisation: an initial particle swarm is randomly distributed within the search space and random "velocities" are assigned.

Step 2 Evaluation: the location of each particle of the swarm (e.g., $x_{i}^{k}=\left[\varepsilon^{\prime} r \tan \delta\right]$, the location of Particle $i$ at Iteration $k$ ) is evaluated using a fitness function, which is defined as a measure of the quality of the solutions produced at each iteration: 
$\mathrm{F}_{\mathrm{PSO}}\left(x_{i}^{k}\right)=\frac{1}{\mathrm{~F}_{\mathrm{err}}\left(x_{i}^{k}\right)+\psi}$

where $\psi$ is a small value to avoid singularity.

This objective function needs to be maximised, and the limit is $1 / \psi$.

Step 3 Velocity vector updating: the velocity vector used to update the current position of each particle is calculated by [26]:

$v_{i}^{k+1}=w v_{i}^{k}+c_{1} r_{1}\left(p_{i}^{k}-x_{i}^{k}\right) / \Delta t+c_{2} r_{2}\left(p_{g}^{k}-x_{i}^{k}\right) / \Delta t$

where $w$ is the inertia weight factor. $v_{i}^{k}$ and $v_{i}^{k+1}$ are the velocities of Particle $i$ at Iterations $k$ and $k+1$, respectively. $r_{1}$ and $r_{2}$ are random numbers between 0 and 1 used to maintain the diversity of the swarm. $p_{i}^{k}$ and $p_{g}^{k}$ are the best position of Particle $i$ (personal best) and the global best position in the swarm within $k$ iterations, respectively. Constant $c_{1}$ represents the cognitive factor that pulls the particle to its own best position. Constant $c_{2}$ is the 'social' factor that pushes the swarm to converge to the current global best position.

The inertia weight factor $w_{k}$ is adjusted dynamically throughout the optimisation process [27]:

$w_{k}=w_{\max }-\frac{w_{\max }-w_{\min }}{k_{\max }-1}(k-1)$

where $w_{\min }$ and $w_{\max }$ are the minimum and maximum inertia weight factors, respectively. $k_{\max }$ is the maximum iteration number.

Step 4 Position updating: the position of Particle $i$ at Iteration $k+1$ is updated by:

$x_{i}^{k+1}=x_{i}^{k}+v_{i}^{k+1} \Delta t$

where $v_{i}^{k+1}$ is the corresponding velocity vector, and $\Delta t$ is the time step value that is set to 1 in the present work.

Step 5 Check convergence: return to Step 2 with $\mathrm{k}=\mathrm{k}+1$. Repeat until the maximum iteration $\mathrm{k}_{\max }$ is met.

\section{Permittivity Calculation for a Ceramic Coated Plate}

\subsection{Forward Calculation}

Here a CST model is built and the values of the reflection coefficient calculated are used as an input for the subsequent inverse calculation of permittivity. As shown in Fig. 3, the model is made up of a waveguide, a dielectric material layer and a metal plate. The X-band microwave frequency range $(8-12 \mathrm{GHz})$ with 401 sampling points is used. The dielectric is a $96 \%$ glazed alumina ceramic, $\varepsilon^{\prime}{ }_{r}=7.2$ and $\tan \delta=$

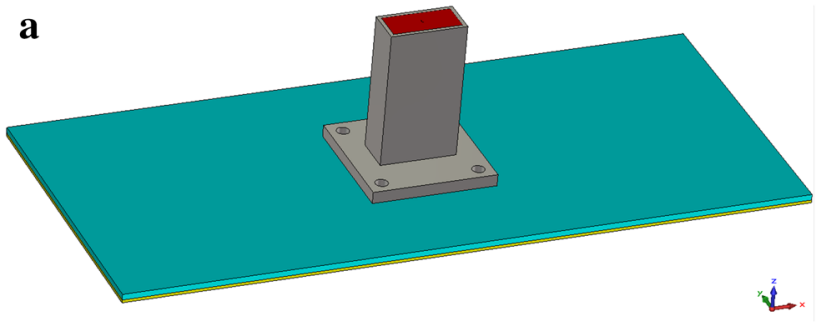

b

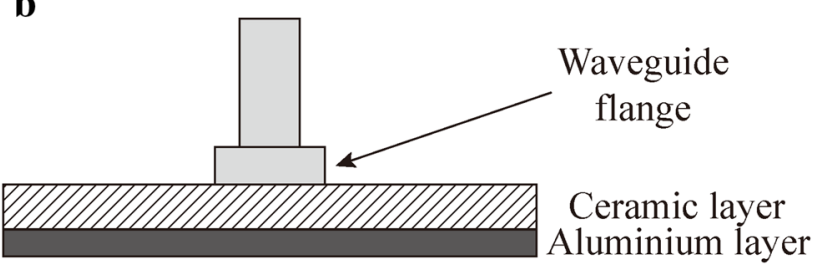

Fig. 3 CST model used to perform the proposed methodology: a 3D view, b side view (not to scale)

0.008 [28], while the metal plate is aluminium, the conductivity of which is directly imported from the built-in material library in CST (i.e., $\sigma=3.56 \times 10^{7} \mathrm{~S} / \mathrm{m}$ ). The thicknesses of the ceramic and aluminium layer are $2 \mathrm{~mm}$ and $1 \mathrm{~mm}$, respectively. The dimensions of the rectangular waveguide flange, ceramic and aluminium layers are given in Table 1. For the given waveguide size and operating frequency range, the allowable maximum standoff distance $d$ is $3.4 \mathrm{~cm}$. In the present case, $d$ is set to zero for simplicity. The inside dimensions of the waveguide are denoted by $\mathrm{a} \times \mathrm{b}$. The ceramic and aluminium layers are of the same planar dimensions, which are set to $\xi \mathrm{a} \times \xi \mathrm{b}$. Here the size factor $\xi$ is defined as the ratio of the model size to the inner dimensions of the waveguide. In the present case, a size factor of 10 is employed. The time domain solver is used to calculate the magnitude and phase of $S_{11}$, which are shown in Fig. 4.

\subsection{Inverse Calculation}

Main parameters of the quasi-Newton method and PSO used for the inverse calculation are listed in Tables 2 and 3, respectively. A high-performance computer (HPC) with an Intel Xeon Central Processing Unit (CPU) and 96 GB memory were used. The simulation results provided by the two optimisation methods are presented in Table 4. The errors of $\varepsilon_{\mathrm{r}}^{\prime}$ given by both methods are within $0.1 \%$, while the error of $\tan \delta$ predicted by both methods is almost the same (3.75\%).

The history of the objective functions, $\mathrm{F}_{\text {err }}$ and FPSO, is presented in Fig. 5. It is seen that the quasi-Newton method converges rapidly requiring only 10 iterations, while for PSO a sharp increase of the fitness function value occurs at the end of the optimisation process, 50 iterations. The maximum fitness function value is $\mathrm{FPSO}_{\mathrm{PSO}}=12.15$, which corresponds to 
Table 1 Dimensions of the waveguide, ceramic layer and aluminium layer (unit: $\mathrm{mm}$ )

\begin{tabular}{|c|c|c|}
\hline Inside dimensions of the waveguide & Outside dimensions of the waveguide & Dimensions of the flange \\
\hline $22.86 \times 10.16$ & $25.40 \times 12.70$ & $41.60 \times 41.60 \times 4.00$ \\
\hline Planar dimensions of ceramic layer & Waveguide lengt & \\
\hline $228.6 \times 101.6$ & 5.00 & \\
\hline
\end{tabular}

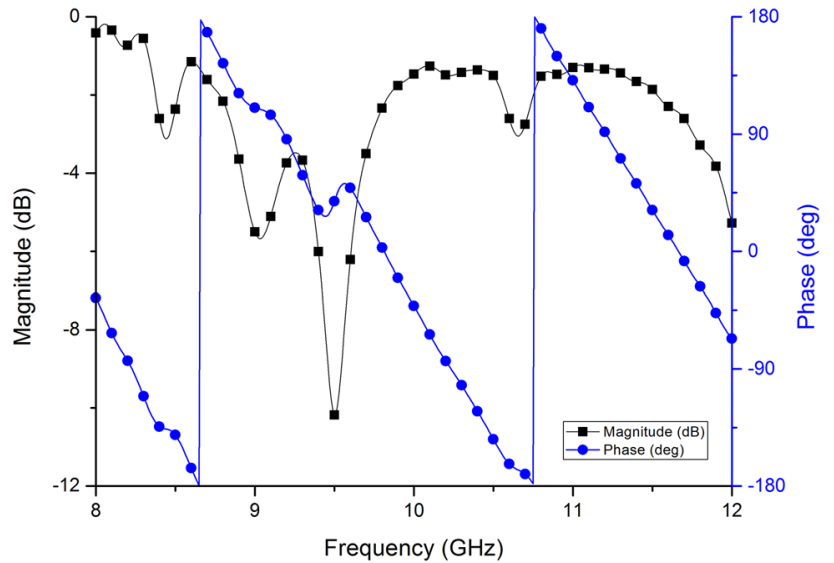

Fig. $4 \mathrm{~S}_{11}$ provided by the CST simulation with known permittivity of the ceramic

the error function value $\mathrm{F}_{\mathrm{err}}=0.07$ according to Equation (5). However, the minimum error function value obtained by the quasi-Newton method is 0.11 . The PSO method does not converge as expected, more accurate results being obtained through the global search. As shown in Fig. 6, in the quasiNewton case $\varepsilon_{\mathrm{r}}^{\prime}$ starts to stabilise after 11 iterations, and $\tan \delta$ levels off after 13 iterations. This indicates that it is slightly faster to find an approximate value for $\varepsilon_{\mathrm{r}}^{\prime}$ than $\tan \delta$. Compared with the Newton method, the solutions by the PSO method are closer to the real values in the first 10 iterations. In the PSO case $\varepsilon_{\mathrm{r}}^{\prime}$ starts to stabilise within 20 iterations, while the $\tan \delta$ curve varies considerably in the first 40 iterations. The computation time of the PSO was nearly $55.58 \mathrm{~h}$, while it took approximately $33.35 \mathrm{~h}$ to complete the quasi-Newton code.

\subsection{Model Size Effect}

From the simulation point of view, for a small test piece it is straightforward to create an exact model. However, for a large structure, it is practical to use a relatively small model to speed up the computation without affecting the accuracy. Hence, in order to investigate the effect of the model size, the responses in four other cases with the same permittivity but varied size factors $(\xi=1,2,5,15$ and 20) are studied. As shown in Fig. 7, the signal converges with increasing $\xi$, and little difference can be found between the cases with $\xi \geq 10$. The curve for $\xi=2$ is slightly different from the other curves, which could be caused by electromagnetic resonance. The size of the sample modelled at $\xi=2$ (i.e., $45.72 \mathrm{~mm} \times$ $20.32 \mathrm{~mm}$ ) is close to the wavelengths of the incident waves (37.5-25 mm for $8-12 \mathrm{GHz}$ ). The computation time of a single simulation run for $\xi=10,15$ and 20 is approximately 2.2, 4 and $6.5 \mathrm{~min}$, respectively. This suggests the CST model with $\xi=10$ can describe the electromagnetic behaviour of a much larger size structure, hence reducing the calculation time.

It should be noted that the size factor is associated with the inner dimensions of the waveguide, which also relates to the available operating frequency range. With a smaller wavelength (i.e., a higher frequency), a smaller mesh grid should be adopted, which would increase the computation time but improve the computational accuracy. Hence, there is a compromise between the frequency and the efficiency.
Table 2 Main parameters of the quasi-Newton method used for the calculation of the permittivity of the ceramic layer

Table 3 Main parameters of the PSO method used for the calculation of the permittivity of the ceramic layer

\begin{tabular}{llll}
\hline Initial estimation of the permittivity & $k_{\max }$ & $k_{\max }^{\prime}$ for 1D line search & $\tau$ \\
\hline$\varepsilon_{\mathrm{r}}^{\prime}=5.0$ and $\tan \delta=0.1$ & 20 & 20 & $10^{-2}$ \\
\hline
\end{tabular}

\begin{tabular}{lllllll}
\hline Population size & $k_{\max }$ & $w_{\min }$ & $w_{\max }$ & $c_{1}$ & $c_{2}$ & $\psi$ \\
\hline 30 & 50 & 0.4 & 0.9 & 2.0 & 2.0 & 0.01 \\
\hline Pre-set search range of $\varepsilon_{\mathrm{r}}^{\prime}$ & & & & Pre-set search range of $\tan \delta$ \\
\hline $1-10$ & & & $0.0001-0.1$ & \\
\hline
\end{tabular}


Table 4 Electric permittivity results obtained by the quasi-Newton and PSO methods

\begin{tabular}{|c|c|c|c|c|c|}
\hline & \multirow[t]{2}{*}{ Glazed alumina ceramic [28] } & \multicolumn{2}{|c|}{ Quasi-Newton method } & \multicolumn{2}{|c|}{ PSO method } \\
\hline & & Value & Error $(\%)$ & Value & Error $(\%)$ \\
\hline$\varepsilon_{\mathrm{r}}^{\prime}$ & 7.2 & 7.2014 & 0.02 & 7.1953 & 0.06 \\
\hline $\tan \delta$ & 0.008 & 0.0077 & 3.75 & 0.0083 & 3.75 \\
\hline
\end{tabular}
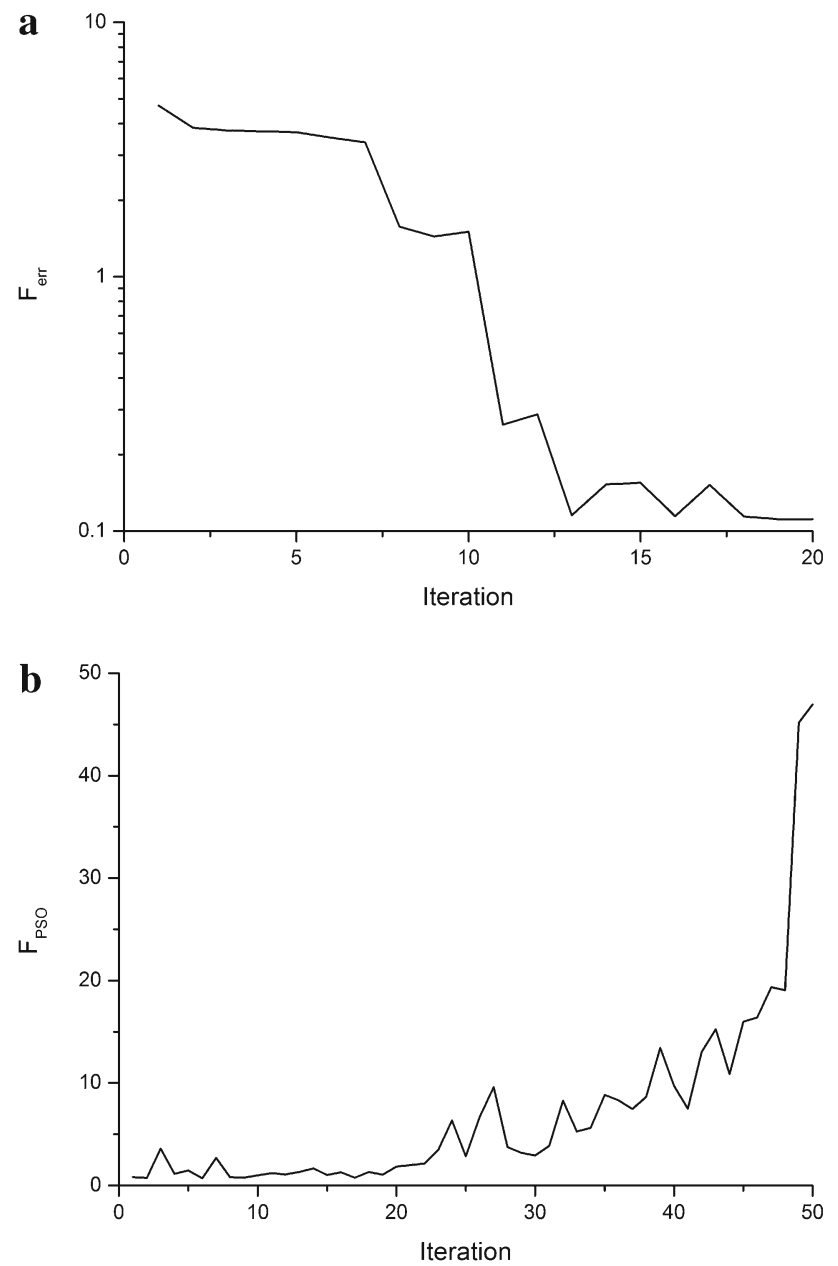

Fig. 5 History of the objective function value in the inverse calculation: a Quasi-Newton method, b PSO method

\subsection{Thickness Calculation}

With the known permittivity, the thickness of the sample layer can be calculated using the CST based methodology. Here 1D Newton-Raphson method is used, since there is only one unknown parameter. The update equation for the thickness parameter $\mathrm{x}$ is:

$x_{k+1}=x_{k}-\mathrm{F}_{\mathrm{err}}\left(x_{k}\right) / \mathrm{F}_{\mathrm{err}}^{\prime}\left(x_{k}\right)$

The process is repeated until the absolute value of the derivative $F_{\text {err }}^{\prime}$ is less than a predefined tolerance. The initial estimation of the thickness for the Newton-Raphson method
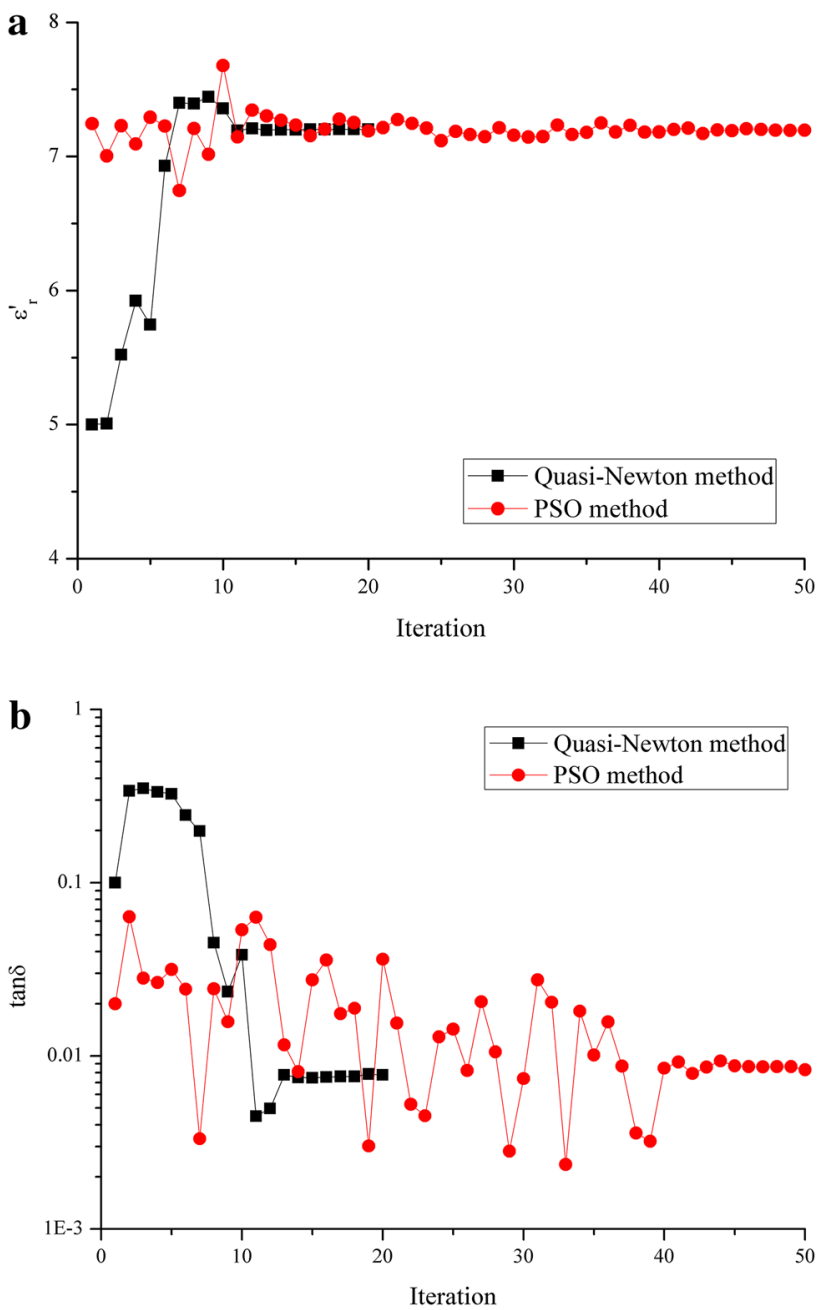

Fig. 6 Variations of $\varepsilon_{r}^{\prime}$ and $\tan \delta$ of the ceramic layer with respect to the iteration: $\mathbf{a} \varepsilon_{r}^{\prime}, \mathbf{b} \tan \delta$

is set to $3 \mathrm{~mm}$, while the pre-set thickness search range for the PSO programme is $0.01-5 \mathrm{~mm}$. The other parameters of the Newton-Raphson and PSO methods are the same as those listed in Tables 2 and 3, respectively. The accurate thickness of $2 \mathrm{~mm}$ is obtained by both methods. The histories of the objective function values and the thickness variable in both cases are presented in Figs. 8 and 9, respectively. In Fig. $8 \mathrm{~b}$, the fitness function value, $\mathrm{F}_{P S O}$, increases as would be expected, since a better solution is generated as it iterates. Similar to the permittivity calculation, the convergence of the Newton-Raphson method is quicker than that of the $\mathrm{PSO}$, and in both cases the solution approaches the actual 

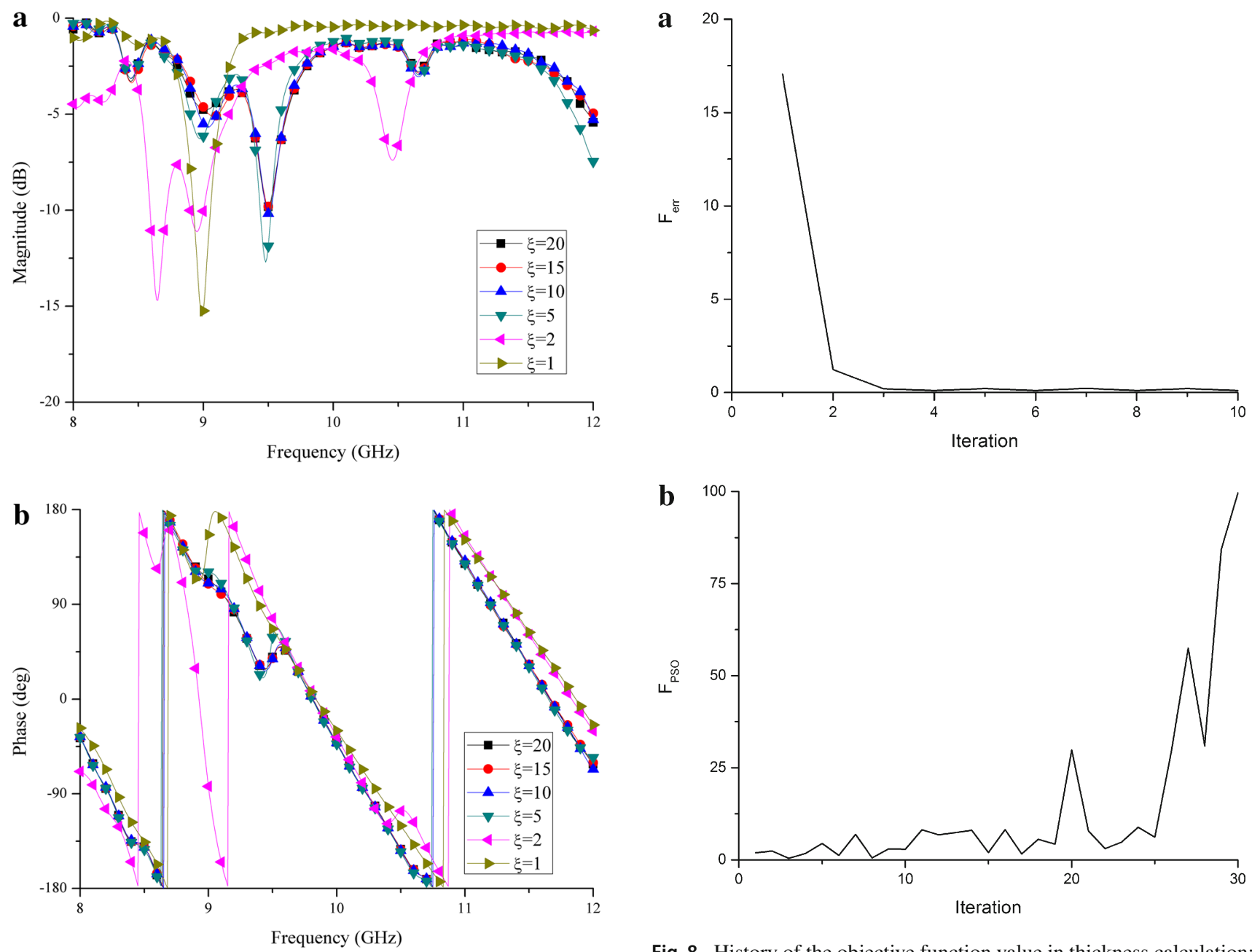

Fig. 7 Comparison of the simulated $\mathrm{S}_{11}$ in the cases with varied size factors of the ceramic layer: a magnitude, $\mathbf{b}$ phase

value after the second iteration. However, to achieve the exact thickness value of $2 \mathrm{~mm}$, the computation time of the PSO programme was roughly $35.10 \mathrm{~h}$ for 30 iterations, while for the Newton-Raphson code it took around 56 min to complete 10 iterations. In this example, the Newton-Raphson method is faster and preferred for estimating the sample thickness.

\section{Test and Permittivity Calculation for a Rogers PCB Laminate}

The permittivity of the dielectric layer in a Rogers ${ }^{\circledR}$ RO4350B PCB laminate with one-sided copper cladding was evaluated. The dielectric was made of a glass woven fabric-reinforced thermoset composite filled with ceramic particles. The length and width of the panel were $304.8 \mathrm{~mm}$ and $228.6 \mathrm{~mm}$, respectively. The thickness of the composite and that of the copper backplate was $1.52 \mathrm{~mm}$ and $35 \mu \mathrm{m}$, respectively.

The permittivity of the composite is available in the datasheet provided by the PCB laminate supplier. It was mea-

Fig. 8 History of the objective function value in thickness calculation: a Quasi-Newton method, b PSO method

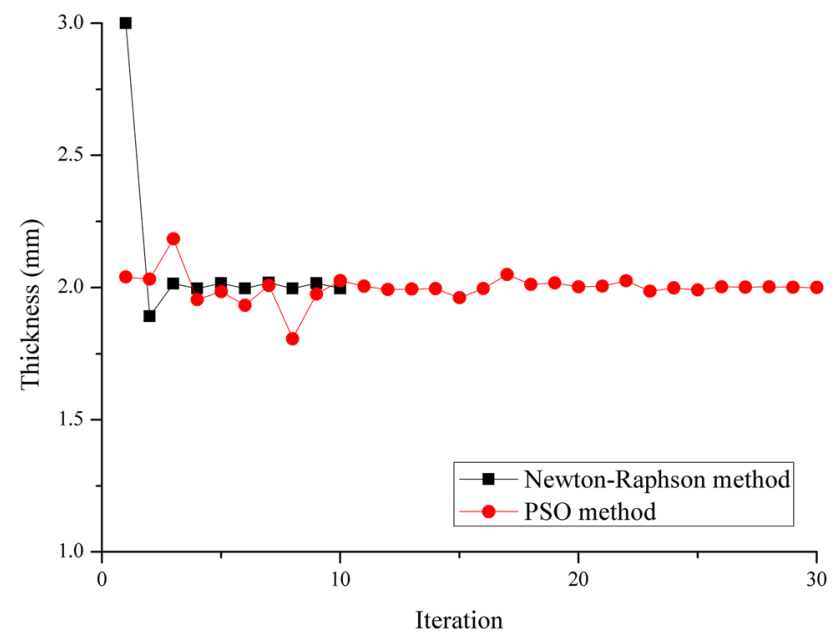

Fig. 9 Variations of the calculated sample thickness with respect to the iteration using the two optimisation methods

sured using the IPC-TM-650 2.5.5.5 standard [29], which is widely adopted in industry. This is a narrowband mea- 
Fig. 10 Transmission line technique for permittivity measurement of the PCB dielectric layer

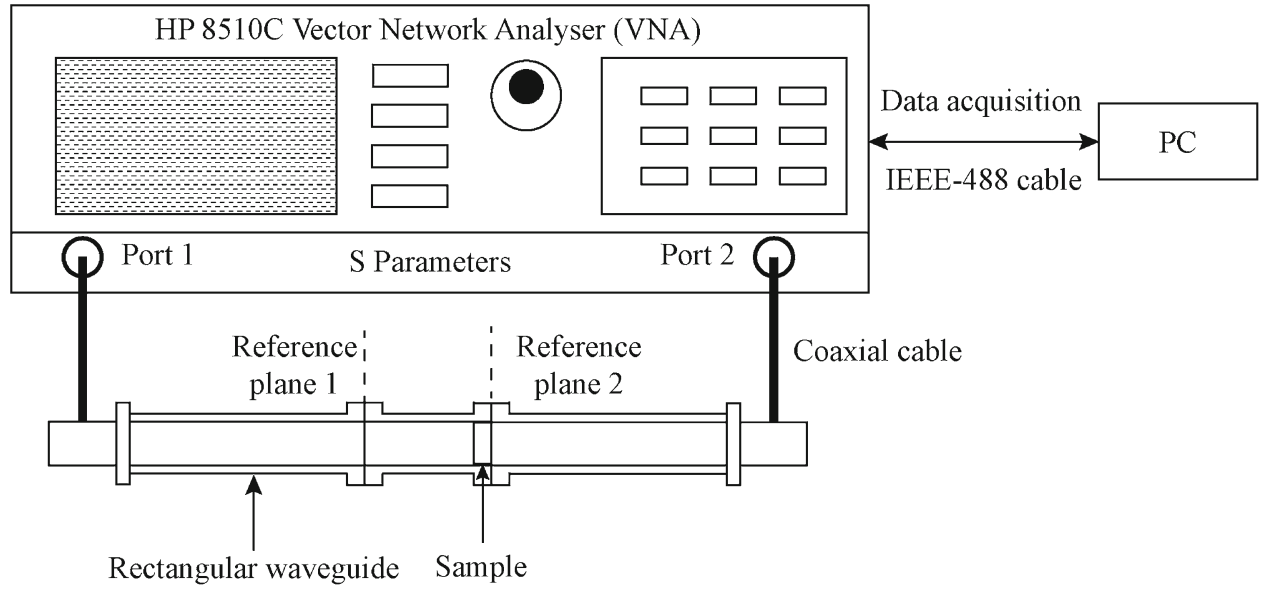

surement, as the permittivity is given at a specific resonance frequency, here of $10 \mathrm{GHz}$ [30].

Two microwave permittivity measurement methods were adopted here: the transmission line broadband technique and the proposed methodology. Like in the IPC test, the material anisotropy due to the woven fibre architecture with respect to the incident electromagnetic field is not considered here but can be accounted for [31].

The setup for the transmission line technique is schematically illustrated in Fig. 10. The VNA was calibrated before the test using the thru-reflect-line (TRL) standard [32]. Calibration accuracy was checked using a flush short (a $6.24 \mathrm{~mm}$ thick copper sheet). Over the operating frequency range, the attenuation was less than $0.1 \mathrm{~dB}$ and the phase shift was $180^{\circ} \pm 0.5^{\circ}$. After the calibration, the S-parameter measurement was carried out at the indicated reference planes, Fig. 10. A personal computer was connected to the VNA by an IEEE-488 cable. A MATLAB programme was developed for data acquisition and permittivity computation. A sample with the inner dimensions of the rectangular waveguide was cut and mounted on the waveguide flange. The transmission coefficients $\left(S_{21}\right)$ were retrieved for permittivity calculation, since this is generally more accurate than using the reflection coefficients $\left(\mathrm{S}_{11}\right)$ [33]. The Nicolson-Ross-Weir algorithm [34] was used for the permittivity calculation. The calculated dielectric constants and loss tangents over $8-12 \mathrm{GHz}$ are presented in Fig. 11. The real part of the permittivity remains relatively stable over the frequency range. Considering the effect of the calibration errors, the uncertainty for the dielectric constant is less than $\pm 0.5 \%$, while for the loss tangent the uncertainty is approximately $\pm 40 \%$.

For the methodology proposed in this work, a single port calibration of the HP8510C VNA used was carried out before the measurement. The measured $S_{11}$ is shown in Fig. 12 . The quasi-Newton method fails to find a reasonable solution, while the PSO method successfully obtains its best solution with a fitness value of 100 at the 8th iteration.

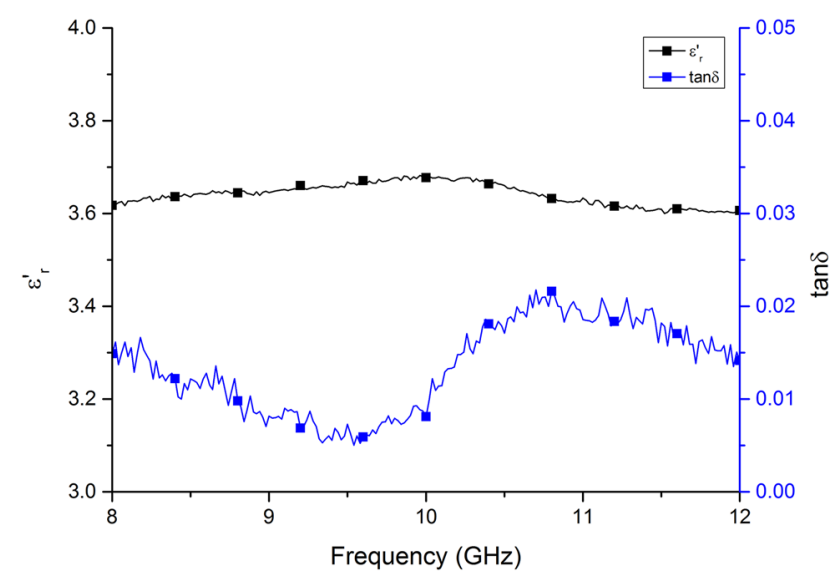

Fig. 11 Permittivity of the PCB dielectric measured using the transmission line technique

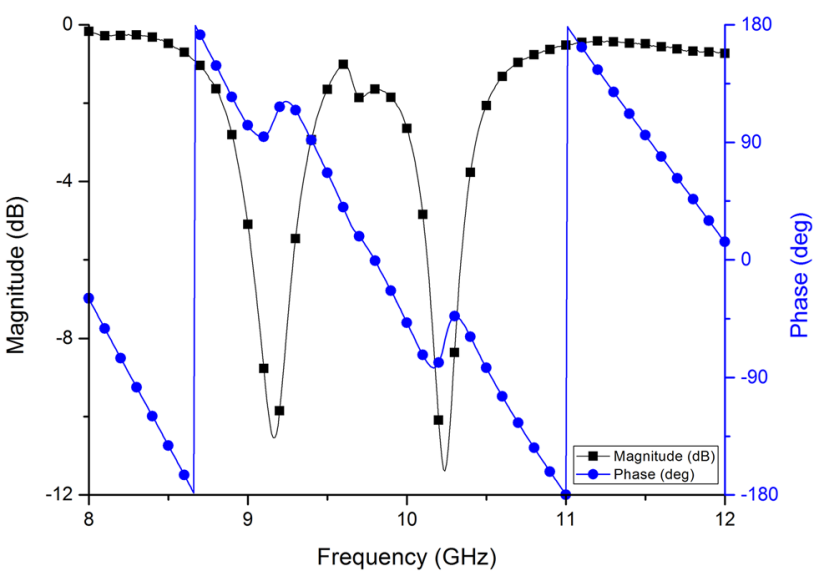

Fig. 12 The magnitude and phase of $S_{11}$ provided by the one-port measurement on the PCB dielectric layer with one-sided copper cladding

The permittivity comparison between the manufacturer data [35] and the measured results is demonstrated in Table 5. There is good agreement in the prediction of the dielectric constant. Due to the low-loss characteristic of the material, 
Table 5 Comparison of the permittivity of the PCB dielectric layer at $10 \mathrm{GHz}$ given by the manufacturer, transmission line technique and the proposed method

\begin{tabular}{lllllll}
\hline & Manufacturer's data [35] & \multicolumn{2}{l}{ Transmission line technique } & & Proposed method & \\
& & Value & Error $(\%)$ & & Value & Error (\%) \\
\hline$\varepsilon_{\mathrm{r}}^{\prime}$ & $3.48( \pm 0.05)$ & $3.68( \pm 0.01)$ & 5.75 & & $3.28( \pm 0.04)$ & 5.74 \\
$\tan \delta$ & 0.0037 & $0.0081( \pm 0.0043)$ & 118.92 & & $0.0037( \pm 0.0003)$ & 0 \\
\hline
\end{tabular}

the loss tangent provided by the transmission line technique is nearly double the manufacturer value. However, the simulation-assisted method proposed offers a more accurate estimation of the loss tangent (within $6 \%$ ).

\section{Concluding Remarks}

A novel non-destructive approach for complex permittivity determination with the use of optimisation and electromagnetic software has been proposed. Using an open-ended waveguide facilitates the measurement process without the need for sample machining, which is relatively operator friendly. In combination with electromagnetic simulation and optimisation, the proposed methodology is readily applied to obtain the electric permittivity $\varepsilon_{\mathrm{r}}$ from the measured reflection coefficient $S_{11}$ data. Two representative optimisation methods (i.e., Newton's method and PSO) have been used. From the simulation results of a ceramic coated plate, it is seen that both methods can offer satisfactory results. The convergence of the Newton method is better than that of the PSO. The PSO can provide approximate solutions without the careful selection of initial guess points required by the Newton method, while its computation process is relatively time-consuming, several hours. Therefore, when choosing a suitable optimisation method, there is a compromise between the accuracy and computation time.

For relatively large test pieces, in order to reduce the computational time, the effect of the CST model size on the microwave signal has been studied. For the same material, little variation of the magnitude and phase is observed when the model is 10 times larger than the inner dimensions of the open-ended rectangular waveguide. In addition, it has been demonstrated that the thickness can also be evaluated using the proposed method when the permittivity is known.

Measurement on a dielectric layer, a woven glass fibrereinforced ceramic filled thermoset material, with a copper cladding was carried out. It has been revealed that the proposed method with the use of PSO can provide more accurate permittivity results than the intrusive in-waveguide measurement. The unsuccessful implementation of the quasi-Newton method is probably due to the computational complexity induced by the two sharp resonance dips, which are observed in the magnitude of $S_{11}$ in Fig. 12.

The computational time is associated with the implementation of the optimisation algorithm, model size, settings of the electromagnetic simulation (e.g., mesh size and solver parameters), performance of the computer used and the material properties to be evaluated. The primary reason for the long computational time demonstrated here is the low loss tangent value of the material used in the example (in the order of $\left.10^{-3}\right)$. It is known that it is more difficult to obtain an accurate measurement of the loss tangent than that of the real part of the permittivity. However, if the results obtained after a few iterations are sufficient for applications, the computational time can be greatly reduced. For the thickness calculation, it is seen that the code can be executed within $1 \mathrm{~h}$ with an acceptable value. Future work is required to improve the computational efficiency, e.g., use of an efficient optimisation method and a high-speed computer.

Acknowledgements This work was financially supported by Dean's Doctoral Scholar Award, School of Materials, The University of Manchester. The first author gratefully acknowledges Colin MacDonald from Rogers (Northern Europe) for providing the PCB laminate sample essential for the experiments.

Open Access This article is distributed under the terms of the Creative Commons Attribution 4.0 International License (http://creativecomm ons.org/licenses/by/4.0/), which permits unrestricted use, distribution, and reproduction in any medium, provided you give appropriate credit to the original author(s) and the source, provide a link to the Creative Commons license, and indicate if changes were made.

\section{References}

1. Zoughi, R., Ganchev, S.: Microwave Nondestructive Evaluation: State-of-the-Art Review. Austin, Texas (1995)

2. Yang, Y., He, C., Wu, B.: Non-destructive microwave evaluation of plasma sprayed TBCs porosity. NDT E Int. 59, 34-39 (2013)

3. Haigh, A.D., Gibson, A.A.P., Chua, H.S., Thompson, F.: Measurement of the wet-mass, moisture content, volume and density of single wheat grain kernels. IEE Proc. Sci. Meas. Technol. 151, 384-388 (2004)

4. Qaddoumi, N., Shroyer, A., Zoughi, R.: Microwave detection of rust under paint and composite laminates. Res. Nondestruct. Eval. 9, 201-212 (1997)

5. Zoughi, R., Kharkovsky, S.: Microwave and millimetre wave sensors for crack detection. Fatigue Fract. Eng. Mater. Struct. 31, 695-713 (2008)

6. Yeh, C., Zoughi, R.: Microwave detection of finite surface cracks in metals using rectangular waveguides. Res. Nondestruct. Eval. 6, 35-55 (1994)

7. Guorong, S., Tianting, Y., Cunfu, H., Shen, Y., Yan, L., Bin, W.: Detection of surface crack on the substrate under thermal barrier coatings using microwave non-destructive evaluation. J. Microw. Power Electromagn. Energy. 49, 69-75 (2015) 
8. Sayar, M., Seo, D., Ogawa, K.: Non-destructive microwave detection of layer thickness in degraded thermal barrier coatings using K- and W-band frequency range. NDT E Int. 42, 398-403 (2009)

9. Li, Z., Haigh, A., Soutis, C., Gibson, A., Sloan, R., Karimian, N.: Delamination detection in composite T-joints of wind turbine blades using microwaves. Adv. Compos. Lett. 25, 83-86 (2016)

10. Li, Z., Haigh, A., Soutis, C., Gibson, A., Sloan, R.: Microwaves sensor for wind turbine blade inspection. Appl. Compos. Mater. 24, 495-512 (2017)

11. Li, Z., Haigh, A.D., Saleh, M.N., McCarthy, E.D., Soutis, C., Gibson, A.A.P., Sloan, R.: Detection of impact damage in carbon fiber composites using an electromagnetic sensor. Res. Nondestruct. Eval., 1-20 (2016)

12. Yang, S.-H., Kim, K.-B., Oh, H.G., Kang, J.-S.: Non-contact detection of impact damage in CFRP composites using millimeter-wave reflection and considering carbon fiber direction. NDT\&E Int. 57, 45-51 (2013)

13. Li, Z., Haigh, A., Soutis, C., Gibson, A., Sloan, R.: Applications of microwave techniques for aerospace composites. In: IEEE Conference on Microwaves, Communications, Antennas and Electronic Systems, Tel Aviv, Israel (2017)

14. Li, Z.: Radio frequency non-destructive evaluation of impact damage in carbon fibre composites (2017)

15. Kraszewski, A.W., Nelson, S.O.: Observations on resonant cavity perturbation by dielectric objects. IEEE Trans. Microw. Theory Tech. 40, 151-155 (1992)

16. Krupka, J.: Frequency domain complex permittivity measurements at microwave frequencies. Meas. Sci. Technol. 17, R55-R70 (2006)

17. Muhammad Firdaus, A.J.K., Sloan, R., Duff, C.I., Wielgat, M., Knowles, J.F.: Nondestructive testing of thermal barrier coated turbine blades using microwave techniques. Mater. Eval. 74, 543-551 (2016)

18. Zoughi, R.: Microwave Non-destructive Testing and Evaluation. Springer, Dordrecht (2000)

19. Bakhtiari, S., Qaddoumi, N., Ganchev, S.I., Zoughi, R.: Microwave noncontact examination of disbond and thickness variation in stratified composite media. IEEE Trans. Microw. Theory Tech. 42, 389-395 (1994)

20. Bakhtiari, S., Ganchev, S., Zoughi, R.: A general formulation for admittance of an open-ended rectangular waveguide radiating into stratified dielectrics. Res. Nondestruct. Eval. 7, 75-87 (1995)

21. Stewart, J.W., Havrilla, M.J.: Electromagnetic characterization of a magnetic material using an open-ended waveguide probe and a rigorous full-wave multimode model. J. Electromagn. Waves Appl. 20, 2037-2052 (2006)
22. CST Microwave Studio 2014. CST Computer Simulation Technology

23. Balanis, C.A.: Antenna Theory: Analysis and Design. Wiley, Hoboken (2012)

24. Nocedal, J., Wright, S.: Numerical Optimization. Springer, New York (1999)

25. Kennedy, J.F., Eberhart, R.C., Shi, Y.: Swarm Intelligence. Morgan Kaufmann Publishers, San Francisco (2001)

26. Shi, Y., Eberhart, R.: A modified particle swarm optimizer. In: 1998 IEEE International Conference on Evolutionary Computation Proceedings. IEEE World Congress on Computational Intelligence (Cat. No. 98TH8360). pp. 69-73. IEEE (1998)

27. Hart, C.G., Vlahopoulos, N.: An integrated multidisciplinary particle swarm optimization approach to conceptual ship design. Struct. Multidiscip. Optim. 41, 481-494 (2010)

28. Robinson, E.A.: Establishment of Quality, Reliability and Design Standards for Low, Medium, and High Power Microwave Hybrid Microcircuits. Texas Instruments, Inc., Dallas (1973)

29. IPC TM-650 2.5.5.5: Stripline Test for Permittivity and Loss Tangent at X-Band (1998)

30. Coonrod, J.: Understanding the variables of dielectric constant for PCB materials used at microwave frequencies. In: 41st European Microwave Conference (EuMC). IEEE, Manchester (2011)

31. Li, Z., Haigh, A., Soutis, C., Gibson, A., Sloan, R.: Dielectric constant of a three-dimensional woven glass fibre composite: analysis and measurement. Compos. Struct. 180, 853-861 (2017)

32. Haigh, A.D., Thompson, F., Gibson, A.A.P., Campbell, G.M., Fang, C.: Complex permittivity of liquid and granular materials using waveguide cells. Subsurf. Sens. Technol. Appl. 2, 425-434 (2001)

33. Li, Z., Haigh, A., Soutis, C., Gibson, A., Sloan, R.: Evaluation of water content in honey using microwave transmission line technique. J. Food Eng. 215, 113-125 (2017)

34. Weir, W.B.: Automatic measurement of complex dielectric constant and permeability at microwave frequencies. Proc. IEEE. 62, 33-36 (1974)

35. RO4000 Series High Frequency Circuit Materials, Connecticut, United States (2015) 